\title{
Evaluating Relevance of Educational Resources of Social and Semantic Web
}

\author{
Davide Taibi ${ }^{1}$, Giovanni Fulantelli ${ }^{1}$, Stefan Dietze $^{2}$, and Besnik Fetahu ${ }^{2}$ \\ ${ }^{1}$ Istituto per le Tecnologie Didattiche, Consiglio Nazionale delle Ricerche, Palermo, Italy \\ \{davide.taibi,giovanni.fulantelli\}@itd.cnr.it \\ ${ }^{2}$ L3S Research Center, Hannover, Germany \\ \{dietze, fetahu\} @13s.de
}

\begin{abstract}
The social web paradigm has modified the way people behave on the Web. Amongst the many consequences of this change the amount of online resources directly produced and shared by users has increased considerably. In this scenario the importance of methods to evaluate the educational relevance of the resources raises up. In this poster we propose an approach based on recent advancements of Linked Open Data.
\end{abstract}

Keywords: Linked Open Data, OER, Educational relevance of resource.

\section{Evaluating Educational Relevance: A Linked Data Approach}

In the last few years the development of the Social Web has changed the way Open Educational Resource repositories are considered, from static collectors of resources to dynamic and social environments able to support social activities such as sharing of knowledge, commenting and voting. At the same time, social media environments, such as YouTube, Flickr, Slideshare, have included specific sections related to education, thus becoming a meaningful source of educational resources. In this scenario the problem of promoting an automated approach to analyze and evaluate the huge amount of resources suitable for learning raised up. Duval and Ochoa have studied relevance ranking metrics for Learning Objects (LOs), proposing the LearnRank function [1] based on Pagerank (the well-known algorithm used by search engines to evaluate the relevance of web pages) and a model to transform the relevance concept into a numerical value [2]. Both authors consider relevance of LOs as a multidimensional issue, where the learning context plays a key role. While these studies focus on the relevance of LOs, the recent evolution of Social Web provides further potentials to infer relevant clues about the educational relevance of resources on social media environments by exploiting social activities such as rating, liking and commenting; furthermore, the concept of learning context is affected as well; finally, the increasing publication of datasets in the background of the Semantic Web requires a review of the previous models to evaluate the educational relevance of resources.

In the framework of the LinkedUp project (http://linkedup-project.eu/), several data curation activities have being carried out, aimed at assessing, cataloging, annotating and exposing all sorts of Web data of educational relevance. Both explicitly 
educational datasets (such as OpenLearn and the mEducator Educational Resources) as well as implicitly educationally relevant datasets (e.g. BBC Programmes and Europeana) have been selected in order to create the Linked Education Catalog. Due to the heterogeneity of the resources selected, a classification of the available datasets, indicating their main purpose, nature and educational relevance is required. Amongst the data curation activities [3][4], in [4] the authors presented an approach, based on a set of data processing techniques to enable resource classification by representing relationships between resources as a network and using cluster algorithms. In this poster the proposed approach has been extended, in order to exploit the properties of the network, such as degree, centrality measures, to provide a relevance measure for learning resources. Moreover, votes, comments, number of visualizations, and other social activities related to each resource can also be used to weight the relationships between resources, thus yielding relevance index that takes into account both social and semantic aspects. Figure 1 shows a part of the resulting network including example resources from BBC, Linked Universities and Europeana datasets.

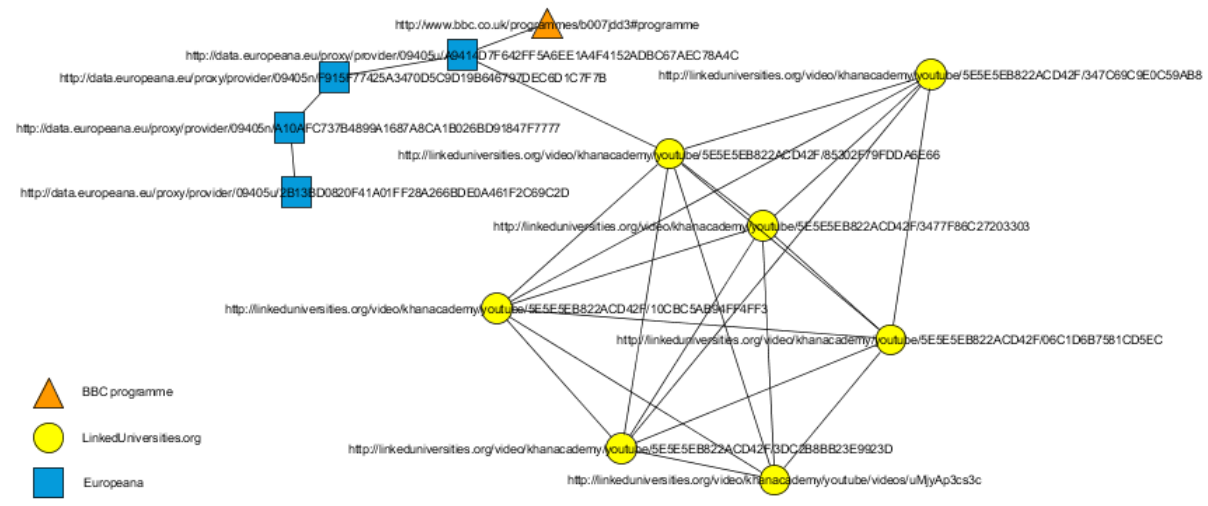

Fig. 1. A network of learning resources

\section{References}

1. Duval, E.: Policy and Innovation in Education - Quality Criteria, chapter LearnRank: the Real Quality Measure for Learning Materials. European Schoolnet, 457-463 (2005)

2. Ochoa, X.: Learnometrics: metrics for learning objects. In: Proc. of the 1st Int. Conf. on Learning Analytics and Knowledge (LAK 2011), pp. 1-8. ACM, New York (2011)

3. d'Aquin, M., Adamou, A., Dietze, S.: Assessing the Educational Linked Data Landscape. In: ACM Web Science (WebSci 2013), Paris, France (May 2013)

4. Taibi, D., Fetahu, B., Dietze, S.: Towards Integration of Web Data into a coherent Educational Data Graph. In: ACM Proceedings of Linked Learning 2013: the 3rd International Workshop on eLearning Approaches for the Linked Data Age (2013) 